\title{
Copy number gain of MYCN gene is a recurrent genetic aberration and favorable prognostic factor in Chinese pediatric neuroblastoma patients
}

Miao Wang ${ }^{1}$, Chunju Zhou ${ }^{2}$, Rongqin Cai ${ }^{1}$, Yong Li $^{1}$ and Liping Gong ${ }^{1 *}$

\begin{abstract}
Background: Amplification of MYCN oncogene is an established marker indicating aggressive tumor progression of neuroblastoma (NBL). But copy number analyses of MYCN gene in ganglioneuroblastoma (GNBL) and ganglioneuroma(GN) is poorly described in the literature. In the study, we evaluated the copy number aberrations of MYCN gene in clinical samples of NBLs, GNBLs and GNs and analyzed their association with clinical outcome of the patients.
\end{abstract}

Methods: In this study, we analyzed MYCN gene and chromosome 2 aneusomy by using fluorescence in situ hybridization (FISH) method in a total of 220 patients with NBL, GNBL and GN cases. Kaplan-Meier curves were generated by using SPSS 12.0 software.

Results: Of 220 patients, 178 (81.0\%) were NBLs, 32 (14.5\%) were GNBLs and $10(4.5 \%)$ were GNs. MYCN gain is a recurrent genetic aberration of neuroblastic tumors $(71.8 \%, 158 / 220)$, which was found in 129 NBLs $(58.6 \%$, 129/220), 25 GNBLs (11.4\%, 25/220) and 4 GN cases (1.8\%, 4/220). However, MYCN amplification was only present in 24 NBL tumors $(13.5 \%, 24 / 178)$ and 1 GNBL case $(3.1 \%, 1 / 32)$. Kaplan-Meier survival analysis indicated that MYCN amplification is significantly correlated with decreased overall survival in NBLs ( $P=0.017)$. Furthermore, a better prognosis trend was observed in patients with MYCN gain tumors compared with those with MYCN gene normal copy number tumors and MYCN amplification tumors ( $P=0.012)$.

Conclusions: In summary, the frequency of MYCN amplification in NBLs is high and is rarely observed in GNBLs and GNs, which suggest MYCN plays an important role in neuroblastic tumors differentiation. MYCN gain appeared to define a subgroup of NBLs with much better outcome and classification of MYCN gene copy number alteration as three groups (amplification, gain and normal) can provide a powerful prognostic indicator in NBLs.

Virtual Slides: The virtual slide(s) for this article can be found here: http://www.diagnosticpathology.diagnomx.eu/ vs/6417541528559124

\footnotetext{
* Correspondence: Ipgong@ccmu.edu.cn

'Department of Pathology, Basic Medical College, Capital Medical University, Beijing, China

Full list of author information is available at the end of the article
} 


\section{Background}

Peripheral neuroblastic tumors (NTs) including neuroblastoma(NBL), ganglioneuroblastoma(GNBL), and ganglioneuroma(GN) comprise one of the most common groups of neoplastic disease in infants and children. NBL and GNBL are considered malignant. In contrast, GNs are considered as benign tumors. In histology, NBL, GNBL, and GN can be conceptualized as three maturational manifestations of a common neoplasm [1].

Amplification of $M Y C N$ oncogene is an established marker indicating aggressive tumor progression of NBL $[2,3]$. Brodeur et al. [4] were the first to show that $M Y C N$ amplification occurs in a substantial subset of primary untreated NBLs and is highly correlated with advanced stage. Seeger et al. [5,6] then demonstrated a strong association with rapid disease progression and a poor prognosis. Analysis of $M Y C N$ remains an essential component of disease evaluation for newly diagnosed NBL patients and serves as a paradigm for the utility of molecular biologic information in cancer treatment stratification [7-9]. $M Y C N$ is vital for proliferation, migration and stem cell homeostasis while decreased levels are associated with terminal neuronal differentiation [10]. On the other hand, downregulation of $M Y C N$ leads to decreased proliferation and differentiation, emphasizing the importance of MYC signaling in NBL biology [11,12]. But copy number status of $M Y C N$ gene in GNBL and GN is poorly described in the literature [13].

In the study, we evaluated the copy number aberrations of MYCN gene in formalin-fixed, paraffin-embedded clinical samples of NBLs, GNBLs and GNs and analyzed their association with clinical outcome of the patients.

\section{Methods}

\section{Tumor tissue and patient information}

Formalin-fixed, paraffin-embedded clinical samples taken from 220 pediatric neuroblastic tumors enrolled on therapeutic or nontherapeutic protocols between 2009 and 2011. Specimen was limited to patients whose diagnosis of neuroblastic tumors was based on histologic and immunohistochemistry examination. Selected clinical and laboratory data (e.g., age at diagnosis, sex, tumor site) were retrieved from the Beijing Children Hospital. The patient characteristics are described in Table 1.

\section{Fluorescence in situ hybridization (FISH)}

$M Y C N$ gene was investigated by interphase FISH on paraffin sections as previously described [14]. Briefly, the $4 \mu \mathrm{m}$-thick tissue sections were deparaffinized and pressure-cooked in $1 \mathrm{mM}$ ethylene diamine tetraacetic acid (EDTA) buffer for $3 \mathrm{~min}$. The tissues were then digested in $0.1 \%$ pepsin solution at $37^{\circ} \mathrm{C}$ for $20 \mathrm{~min}$, dehydrated and added with the appropriate probes. $M Y C N$ SG/CEP2 SO probe is used in this study (Vysis, Abbott Laboratories, Abbott Park, IL). The slides were
Table 1 Patient characteristics

\begin{tabular}{lccc}
\hline Characteristics & GN & GNBL & NBL \\
\hline Patients [no. \% ] & 10 & 32 & 178 \\
Median age (years) & 3 & 3.5 & 1.8 \\
Male/female ratio & $4 / 6$ & $14 / 18$ & $113 / 65$ \\
Sites & & & \\
adrenals & 1 & 12 & 67 \\
thorax & 6 & 12 & 31 \\
abdomen & 2 & 8 & 50 \\
pelvis & 0 & 0 & 10 \\
others & 1 & 0 & 20 \\
MYCN status & & & \\
normal & 6 & 6 & 25 \\
gain & 4 & 25 & 129 \\
amplification & 0 & 1 & \\
\hline
\end{tabular}

GN :Ganglioneuroma; GNBL: Ganglioneuroblastoma; NBL: Neuroblastoma.

incubated at $80^{\circ} \mathrm{C}$ for $25 \mathrm{~min}$ and at $45^{\circ} \mathrm{C}$ for 2 days. The slides were then washed in post-hybridization buffers, stained with anti-fade solution containing 4',6diamidino-2-phenylindole (DAPI; Vector Labs, Burlingame, CA) and examined using a fluorescence microscope (BX51; Olympus, Tokyo, Japan) by two investigators independently. Slides with known structural or numerical abnormality for the above probes were used as positive controls, and a case of reactive hyperplasia of the tonsil was used as a negative control.

\section{FISH scoring scheme}

Fluorescence microscopy was performed with a BX51 microscope equipped with filter set for FITC, Texas red, and DAPI. Each sample was analyzed to determine the origin of the amplification unit (extrachromosomal double minutes or intrachromosomal homogeneously staining regions) and the proportion of cells with amplified MYCN genes. The FISH signals were scored in 200 no overlapping nuclei per core, independently by two investigators (M Wang and LP Gong.), and the consensus was recorded. Four cellular groups were defined as previous study [15]. No Alteration: cells with $2 \mathrm{MYCN}$ signals and 2 CEP2 signals; Amplification: The number of $M Y C N$ signals is at least 10 copies greater than the control probe signals;Loss/Imbalance:Presence of at least 2 MYCN signals and increased CEP2 signals;Gain: The number of MYCN signals is 1-9 copies more than CEP2 signals.

\section{Statistical analysis}

SPSS 12.0 software (SPSS, Chicago, IL) was used to analyze differences in neuroblastic tumor characteristics among the patient groups. The $x^{2}$ test or two-tailed Fisher's exact test was used to compare the predictive values of each 
marker analyzed. A p value of less than 0.05 was considered statistically significant. Kaplan-Meier curves were generated by using SPSS 12.0 software.

\section{Results}

\section{Clinical features}

We analyzed a panel of 220 pediatric patient samples comprising 178 NBLs, 32 GNBLs, and 10 GNs. Patient characteristics are summarized in Table 1 . There was a male predominance in NBL disease group, with the male to female ratio being 1.7:1. The median age at diagnosis was 3 years for GNs, 3.5 years for GNBLs and 1.8 years for NBLs. Patients with NBL were significantly younger than patients with GN and GNBL $(\mathrm{P}<0.001)$. The distribution of these three diseases generally follows the distribution of the sympathetic ganglia. In our study, about 36.4\% (80/220) tumors arise in adrenal gland. In addition, adrenal involvement at diagnosis was 1 case for GN, 12 cases for GNBL and 67 cases for NBL.

\section{Morphology features}

Neuroblastic tumors were divided into three histological subtypes on morphologic criteria of neuroblastic tumors which was recommendations by the International Neuroblastoma Pathology Committee [16]. GN, a fully differentiated tumor, is characterized by a mixture of mature schwann cells and ganglion cells (Figure 1A). GNBL has primitive neuroblasts along with maturing ganglion cells (Figure 1B); the number and arrangement of the cells vary so the tumor assumes a wide range of appearances. NBL, the least differentiated, resembles the fetal adrenal medulla and is composed of primitive neuroblasts (Figure 1C).
GNBLs and GNs are usually of favorable histology. In fact, GN is considered a benign neuroblastic tumor.

\section{MYCN gene status analysis}

$M Y C N$ gene FISH analysis was successful in all cases of GN, GNBL and NBL, and the results are detailed in Table 1. MYCN status was determined in light of chromosome 2 copy number. No cases showed loss of centromere 2 and $M Y C N$. But in total, 158 cases with $M Y C N$ gene gain also showed gain of centromere 2, suggesting polyploidy in these cases.

Among 178 NBL cases, 153 (85.9\%) were MYCN gene alterations, including 24 cases (13.5\%) with MYCN amplification [Figure 2A] and 129 cases (72.5\%) with $M Y C N$ gain [Figure 2B]. MYCN gene normal [Figure 2C] was only found in 25 cases (14.0\%) of NBL, which was significantly lower than in GNBL and in GN $(\mathrm{p}<0.05)$.

In GN and GNBL cases, six of ten (40.0\%) and six of thirty-two (18.8\%) cases showed no aberration of $M Y C N$ gene, respectively. The frequency of $M Y C N$ gain was significantly higher in GNBL $(78.1 \%, 25 / 32)$ than in GN $(40.0 \%, 4 / 10)(\mathrm{p}<0.05)$.

\section{Prognostic analysis}

Sixty-seven NBL cases had follow-up information available, with 50 survived and 17 died at the time of writing up of the study. The patients typically received multiagent chemotherapy. The survival time ranged from 0 to 47 months and the average survival time was 37.4 months. A significant trend was observed between $M Y C N$ gene amplification tumors and poor outcome compared with those with no amplification of $M Y C N$ gene patients $(\mathrm{p}=0.017)$ (Figure 3A). Interestingly,the Kaplan-Meier survival

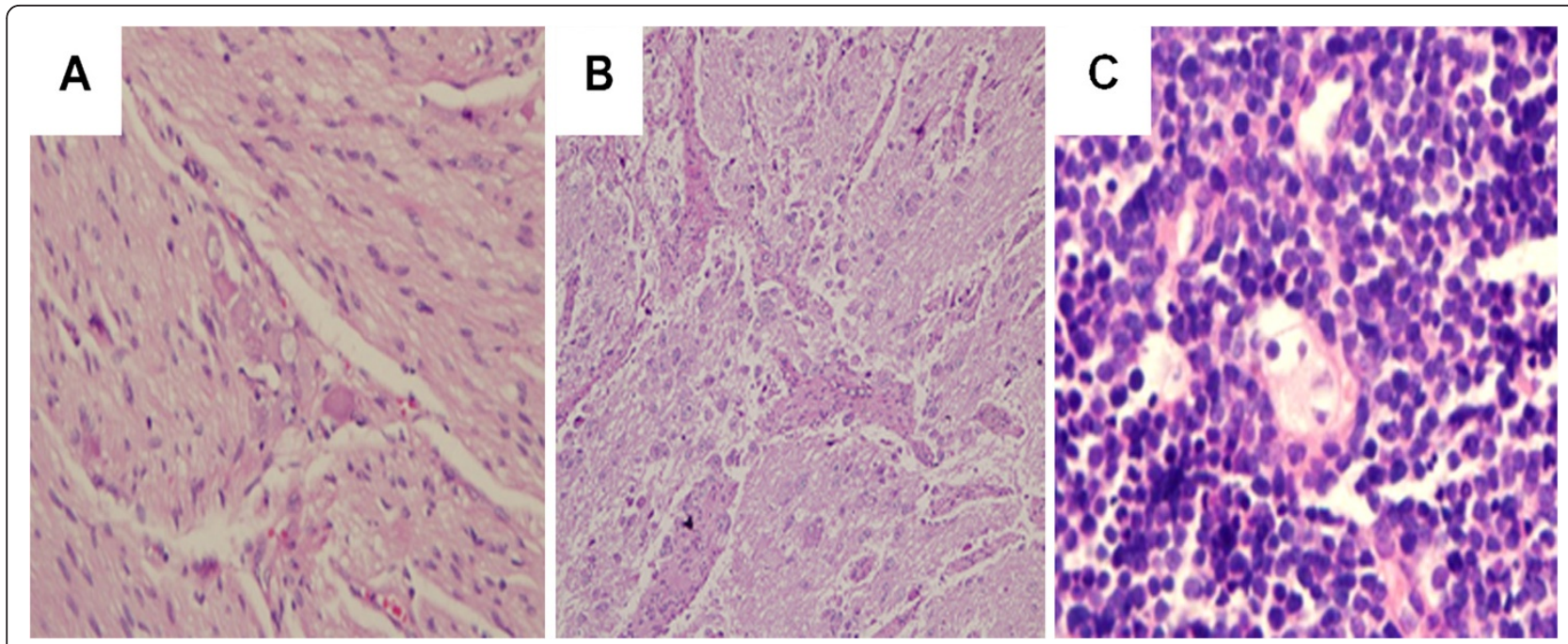

Figure 1 Representative HE image of neuroblastic tumors. A, Ganglioneuroma: Mature ganglion cell with schwannian stroma. B, Ganglioneuroblastoma: Increased schwannian stroma. C, Neuroblastoma: A monotonous population of hyperchromatic cells with scant cytoplasm. 

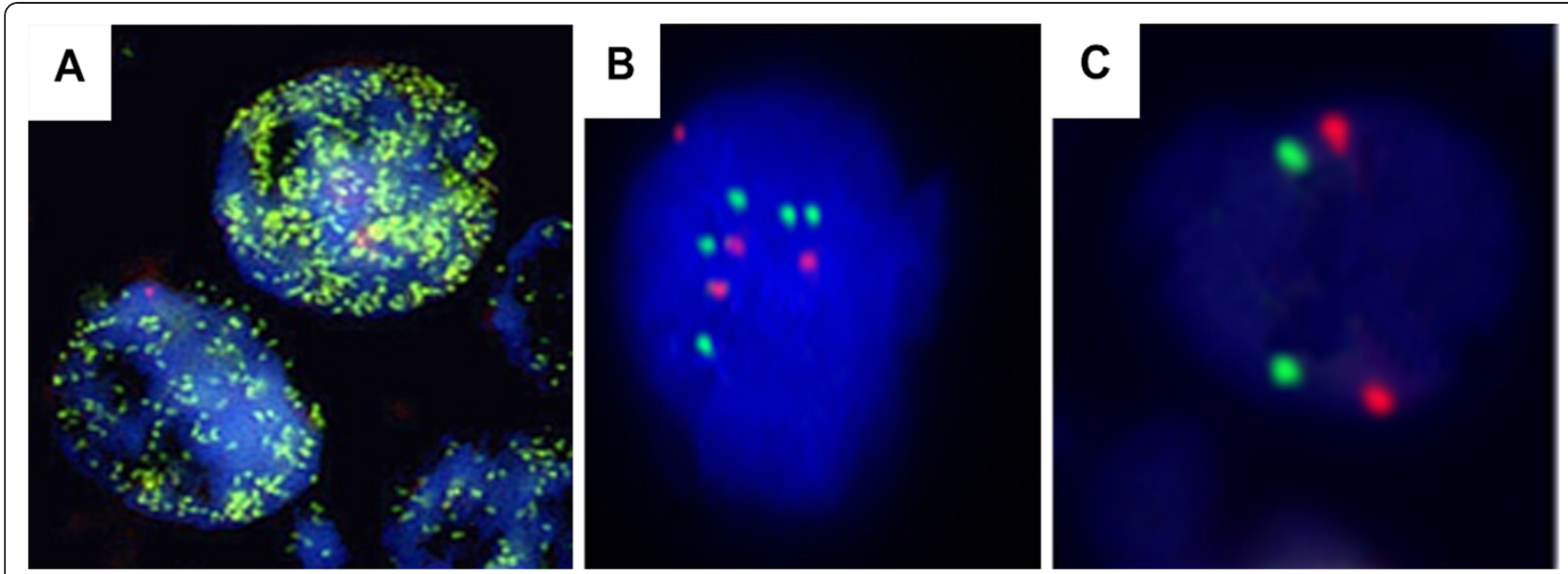

Figure 2 Representative FISH image of neuroblastic tumor cells displaying MYCN gene status. A: Amplification:The number of MYCN signals (green) is more than 10 copies of the CEP2 probe signals (red). B: Gain:The number of MYCN signals (green) is 1 copy greater than the CEP2 probe signals; C: No Alteration: Cells with MYCN signals (green) showing the same numbers of the CEP2 probe signals. (DAPI counterstain, original magnification $\times 1000$ ).

analysis also indicated a significant better prognosis in patients with $M Y C N$ gene gain tumors compared with those with $M Y C N$ gene normal tumors $(\mathrm{p}=0.012)$ (Figure 3B).

\section{Discussion}

The peripheral neuroblastic tumour group includes NBL, GNBL and GN. NBL is the most common extracranial solid tumour of childhood and the incidence of pediatric neuroblastoma are increasing [17,18]. MYCN gene amplification is a known molecular marker for aggressive progression of NBL [4]. In the study, we evaluated the histological presentation and MYCN gene copy number in 220 pediatric neuroblastic tumors, which include 178 NBLs, 32 GNBLs and 10 GNs and analyzed their association with clinical outcome of the patients. To our knowledge, this is the first article for $M Y C N$ gene and chromosome 2 aneusomy analyses by using fluorescence in situ hybridization (FISH) method in chinese pediatric patients.

Our study reaffirmed the need for MYCN copy number to be determined in light of chromosome 2 copy number. MYCN copy number had been determined by southern blot analysis [19]. After 1993, fluorescence in situ hybridization (FISH) was used to determine the presence of MYCN amplification [20,21]. In these studies, the results of southern blotting and FISH analysis were prospectively compared and a $M Y C N$ copy number of $\geq 10$ was determined to be the optimal cutoff by FISH [20], as the vast majority of amplified tumors have very

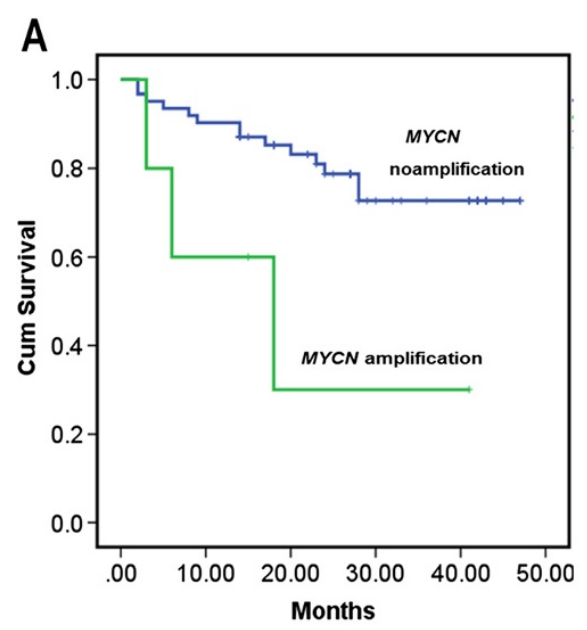

B

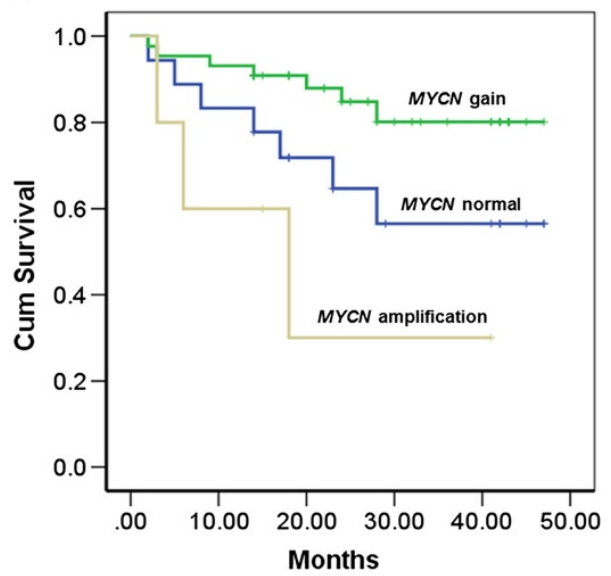

Figure 3 Overall survival analysis. A. MYCN amplification is correlated with decreased overall survival in NBL. P=0.017. B. MYCN amplification is correlated with decreased overall survival and MYCN gene gain is correlated with good outcome in NBL. P=0.012. 
large numbers of double minutes in each tumor cell. By southern blotting, any normal cells in the tissue were included in the measurement, whereas by FISH, each tumor nucleus was visualized directly and simultaneous cohybridization with a specific chromosome probe is of great value in predicting the prognosis of patients [22]. FISH has a higher sensitivity because it detects the $M Y C N$ copy number on the single-cell level and allows correlation of morphologic details. In our estimation, FISH is a practical, useful and reliable method for analysis of $M Y C N$ copy number in neuroblastic tumors.

Our results showed that aberrant $M Y C N$ copy number was detected in 153 (85.9\%) of 178 NBLs, with amplification constituting 24 (13.5\%), gain $129(72.4 \%)$. In contrast, MYCN amplification is only observed in one GNBL case $(1 / 32,3.1 \%)$ and no GN cases $(0 / 10,0 \%)$. Moll A et al. [23] also reported that no amplification of the $M Y C N$-oncogene was found in mixed hepatoblastoma and teratoma of the liver in a 3-year-old boy. Wan, T.S et al. [24] investigated $12 \mathrm{NBL}$ patients for $M Y C N$ amplification by FISH and found that $16.7 \%$ cases had $M Y C N$ amplification. Angelini,P et al. [13] reported that only about $2 \%$ had $M Y C N$ gene amplified tumours in 232 GNBL patients. Our results also showed that the frequency of MYCN gene gain was significantly higher in GNBL $(78.1 \%, 25 / 32)$ and NBL $(72.5 \%, 129 / 178)$ than in GN $(40.0 \%, 4 / 10)$. Toraman,A.D et al. [25] found that chromosomal gains displayed by chromosomes and chromosome loci were 2 p25 approximately pter (60\%) in five GNBL cases by comparative genomic hybridization. Truong LN et al. [26] also detected MYCN oncogenes in malignant brain tumors by using multiplex ligation dependent probe amplification (MLPA). Thus, higher frequency of $M Y C N$ gene aberrations in undifferentiated or less differentiated tumors indicates an important function of $M Y C N$ gene in tumor malignancy.

$M Y C N$ amplification is an established marker indicating aggressive tumor progression of NBL [27]. Our data also showed that $M Y C N$ amplification is correlated with decreased overall survival in NBL $(\mathrm{P}=0.017)$ (Figure $3 \mathrm{~A})$. More significantly, we demonstrated for the first time that the presence of extra copies of MYCN gene is an independent prognostic factor for NBL in our case series. The patients with $M Y C N$ gene gain had a significantly longer mean survival time than those with normal MYCN gene copy number $(\mathrm{P}=0.012)$ (Figure 3B). In our data, NBL cases with $M Y C N$ gene gain also showed gain of centromere 2, suggesting polyploidy in these cases. In 1991, look et al. [28] found that NBL patietns treated with cyclophosphamide-doxorubicin, hyperdiploidy was closely associated with long-term disease-free survival (greater than $90 \%$ of cases), while diploidy invariably predicted early treatment failure $(\mathrm{P}<0.001)$. Recently, George et al. had also found that NB patients with hyperdiploidy plus no amplified $M Y C N$ confers a favorable prognosis [29], which is in line with our study. Furthermore, they also found that hyperdiploidy plus no amplified MYCN NBL patients may respond well to contemporary chemotherapy, and could be spared intensive myeloablative therapy with stem-cell rescue [29]. Thus, the classification of $M Y C N$ gene status as three groups by FISH may provide more powerful prognostic indicator and better treatment options in NBL.

\section{Conclusions}

In summary, using simple and easily applicable FISH technique we showed in the present study that the frequency of MYCN amplification in NBLs is high and is rarely observed in GNBLs and GNs, which suggest MYCN gene play an important role in neuroblastic tumors differentiation. Furthermore, the copy number gain of $M Y C N$ gene locus appeared to define a subgroup of NBL with much better outcome and classification of $M Y C N$ gene copy number alteration as three groups (amplification, gain and normal) can provide a powerful prognostic indicator in NBL.

\section{Abbreviations}

GN: Ganglioneuroma; GNBL: Ganglioneuroblastoma; NBL: Neuroblastoma; FISH: Fluorescence in situ hybridization.

\section{Competing interests}

The authors declare that they have no competing interests.

\section{Authors' contributions}

Miao Wang, Yong Li and Rongqin Cai carried out the FISH studies. Miao Wang drafted the manuscript. Miao Wang, Chunju Zhou and Liping Gong participated in the design of the study. Chunju Zhou participated in and coordinated specimen and clinical data retrieval and characterization. Liping Gong conceived and coordinated the study. All authors read and approved the final manuscript. This study was conducted with approval from Capital Medical University.

\section{Acknowledgements}

This study was funded by Natural Science Foundation of China (81001054), Excellent talents of Science Program, Beijing, China (2011D005018000008), Foundation for University Key Teacher by the Ministry of Education (PHR201108096) with the purpose of encouraging high-value research.

\section{Author details}

'Department of Pathology, Basic Medical College, Capital Medical University, Beijing, China. ${ }^{2}$ Department of Pathology, Beijing Children's Hospital, Capital Medical University, Beijing, China.

Received: 24 November 2012 Accepted: 8 January 2013 Published: 15 January 2013

\section{References}

1. Joshi W, Silverman JF: Pathology of neuroblastic tumors. Semin Diagn Pathol 1994, 11(2):107-117.

2. Schwab M: Amplification of $\mathrm{N}$-myc as a prognostic marker for patients with neuroblastoma. Semin Cancer Biol 1993, 4(1):13-18.

3. Rubie H, Hartmann O, Michon J, Frappaz D, Coze C, Chastagner P, Baranzelli MC, Plantaz D, Avet-Loiseau H, Benard J, et al: N-Myc gene amplification is a major prognostic factor in localized neuroblastoma: results of the French NBL 90 study. Neuroblastoma Study Group of the Societe Francaise d'Oncologie Pediatrique. J Clin Oncol 1997, 15(3):1171-1182. 
4. Brodeur GM, Seeger RC, Schwab M, Varmus HE, Bishop JM: Amplification of $\mathrm{N}$-myc in untreated human neuroblastomas correlates with advanced disease stage. Science 1984, 224(4653):1121-1124.

5. Brodeur GM, Seeger RC, Schwab M, Varmus HE, Bishop JM: Amplification of $\mathrm{N}$-myc sequences in primary human neuroblastomas: correlation with advanced disease stage. Prog Clin Biol Res 1985, 175:105-113.

6. Seeger RC, Brodeur GM, Sather H, Dalton A, Siegel SE, Wong KY, Hammond $\mathrm{D}$ : Association of multiple copies of the $\mathrm{N}$-myc oncogene with rapid progression of neuroblastomas. N Engl J Med 1985, 313(18):1111-1116.

7. Tonini GP, Verdona G, Garaventa A, Cornaglia-Ferraris P: Antiblastic treatment does not affect $\mathrm{N}$-myc gene amplification in neuroblastoma. Anticancer Res 1987, 7(4B):729-732.

8. Cetinkaya C, Hultquist A, Su Y, Wu S, Bahram F, Pahlman S, Guzhova I, Larsson LG: Combined IFN-gamma and retinoic acid treatment targets the N-Myc/Max/Mad1 network resulting in repression of N-Myc target genes in MYCN-amplified neuroblastoma cells. Mol Cancer Ther 2007, 6(10):2634-2641.

9. Pession A, Tonelli R: The MYCN oncogene as a specific and selective drug target for peripheral and central nervous system tumors. Curr Cancer Drug Targets 2005, 5(4):273-283.

10. Rouah E, Wilson DR, Armstrong DL, Darlington GJ: N-myc amplification and neuronal differentiation in human primitive neuroectodermal tumors of the central nervous system. Cancer Res 1989, 49(7):1797-1801.

11. Kang JH, Rychahou PG, Ishola TA, Qiao J, Evers BM, Chung DH: MYCN silencing induces differentiation and apoptosis in human neuroblastoma cells. Biochem Biophys Res Commun 2006, 351(1):192-197.

12. Janardhanan R, Banik NL, Ray SK: N-Myc down regulation induced differentiation, early cell cycle exit, and apoptosis in human malignant neuroblastoma cells having wild type or mutant p53. Biochem Pharmacol 2009, 78(9):1105-1114.

13. Angelini $P$, London WB, Cohn SL, Pearson AD, Matthay KK, Monclair T, Ambros PF, Shimada H, Leuschner I, Peuchmaur M, et al: Characteristics and outcome of patients with ganglioneuroblastoma, nodular subtype: a report from the INRG project. Eur J Cancer 2012, 48(8):1185-1191.

14. Yu R, Chen G, Zhou C, Gao Z, Shi Y, Shi Y, Zhou X, Xie J, Liu H, Gong L: Extra copies of ALK gene locus is a recurrent genetic aberration and favorable prognostic factor in both ALK-positive and ALK-negative anaplastic large cell lymphomas. Leuk Res 2012, 36(9):1141-1146.

15. Ambros PF, Ambros IM: Pathology and biology guidelines for resectable and unresectable neuroblastic tumors and bone marrow examination guidelines. Med Pediatr Oncol 2001, 37(6):492-504.

16. Shimada H, Ambros IM, Dehner LP, Hata J, Joshi W, Roald B: Terminology and morphologic criteria of neuroblastic tumors: recommendations by the International Neuroblastoma Pathology Committee. Cancer 1999, 86(2):349-363.

17. Hsieh MH, Meng MV, Walsh TJ, Matthay KK, Baskin LS: Increasing incidence of neuroblastoma and potentially higher associated mortality of children from nonmetropolitan areas: analysis of the surveillance, epidemiology, and end results database. J Pediatr Hematol Oncol 2009, 31(12):942-946.

18. Heck JE, Ritz B, Hung RJ, Hashibe M, Boffetta P: The epidemiology of neuroblastoma: a review. Paediatr Perinat Epidemiol 2009, 23(2):125-143.

19. Schwab M, Alitalo K, Klempnauer KH, Varmus HE, Bishop JM, Gilbert F, Brodeur G, Goldstein M, Trent J: Amplified DNA with limited homology to myc cellular oncogene is shared by human neuroblastoma cell lines and a neuroblastoma tumour. Nature 1983, 305(5931):245-248.

20. Mathew P, Valentine MB, Bowman LC, Rowe ST, Nash MB, Valentine VA, Cohn SL, Castleberry RP, Brodeur GM, Look AT: Detection of MYCN gene amplification in neuroblastoma by fluorescence in situ hybridization: a pediatric oncology group study. Neoplasia 2001, 3(2):105-109.

21. Shapiro DN, Valentine MB, Rowe ST, Sinclair AE, Sublett JE, Roberts WM, Look AT: Detection of N-myc gene amplification by fluorescence in situ hybridization. Diagnostic utility for neuroblastoma. Am J Pathol 1993, 142(5):1339-1346.

22. Hachitanda Y, Saito M, Mori T, Hamazaki M: Application of fluorescence in situ hybridization to detect N-myc (MYCN) gene amplification on paraffin-embedded tissue sections of neuroblastomas. Med Pediatr Oncol 1997, 29(2):135-138.

23. Moll A, Krenauer A, Bierbach U, Till H, Hirsch W, Leuschner I, Schmitz N, Wittekind C, Aigner T: Mixed hepatoblastoma and teratoma of the liver in a 3-year-old child: a unique combination and clinical challenge. Diagn Pathol 2009, 4:37.
24. Wan TS, Ma ES, Chan GC, Chan LC: Investigation of MYCN status in neuroblastoma by fluorescence in situ hybridization. Int J Mol Med 2004 14(6):981-987

25. Toraman AD, Keser I, Luleci G, Tunali N, Gelen T: Comparative genomic hybridization in ganglioneuroblastomas. Cancer Genet Cytogenet 2002, 132(1):36-40.

26. Truong LN, Patil S, Martin SS, LeBlanc JF, Nanda A, Nordberg ML, Beckner ME: Rapid Detection of high-level oncogene amplifications in ultrasonic surgical aspirations of brain tumors. Diagn Pathol 2012, 7:66.

27. Suita S, Zaizen Y, Kaneko M, Uchino J, Takeda T, Iwafuchi M, Utsumi J, Takahashi H, Yokoyama J, Nishihira H, et al: What is the benefit of aggressive chemotherapy for advanced neuroblastoma with $\mathrm{N}$-myc amplification? A report from the Japanese Study Group for the Treatment of Advanced Neuroblastoma. J Pediatr Surg 1994, 29(6):746-750.

28. Look AT, Hayes FA, Shuster JJ, Douglass EC, Castleberry RP, Bowman LC, Smith El, Brodeur GM: Clinical relevance of tumor cell ploidy and N-myc gene amplification in childhood neuroblastoma: a Pediatric Oncology Group study. J Clin Oncol 1991, 9(4):581-591.

29. George RE, London WB, Cohn SL, Maris JM, Kretschmar C, Diller L, Brodeur GM, Castleberry RP, Look AT: Hyperdiploidy plus nonamplified MYCN confers a favorable prognosis in children 12 to 18 months old with disseminated neuroblastoma: a Pediatric Oncology Group study. J Clin Oncol 2005, 23(27):6466-6473.

doi:10.1186/1746-1596-8-5

Cite this article as: Wang et al: Copy number gain of MYCN gene is a recurrent genetic aberration and favorable prognostic factor in Chinese pediatric neuroblastoma patients. Diagnostic Pathology 2013 8:5.

\section{Submit your next manuscript to BioMed Central and take full advantage of:}

- Convenient online submission

- Thorough peer review

- No space constraints or color figure charges

- Immediate publication on acceptance

- Inclusion in PubMed, CAS, Scopus and Google Scholar

- Research which is freely available for redistribution

Submit your manuscript at www.biomedcentral.com/submit
C) Biomed Central 